\title{
Conhecimento em saúde sexual e reprodutiva: estudo transversal com adolescentes
}

\author{
Patrícia Carvalho Oliveira ${ }^{1}$, Laurena Moreira Pires², Ana Luiza Neto Junqueira ${ }^{3}$, \\ Maria Aparecida da Silva Vieira ${ }^{4}$, Marcos André Matos ${ }^{5}$, Karlla Antonieta Amorim Caetano ${ }^{6}$, \\ Ruth Minamisava7, Sheila Araújo Teles ${ }^{8}$, Márcia Maria de Souza9
}

${ }^{1}$ Enfermeira, Doutora em Ciências da Saúde. Coordenadora dos Programas de Atenção Integral à Saúde do Homem e do Adolescente de Aparecida de Goiânia. Aparecida de Goiânia, GO, Brasil. E-mail: patico.nurse@gmail.com.

2 Enfermeira, Mestre em Enfermagem. Discente do Programa de Pós-Graduação em Enfermagem, nível Doutorado, da Universidade Federal de Goiás. Goiânia, GO, Brasil. E-mail: laurenapires@gmail.com. ${ }^{3}$ Enfermeira, Doutora em Ciências da Saúde. Professora Adjunta da Universidade Federal de Goiás. Goiânia, GO, Brasil. E- mail:

ananeto.fen@gmail.com.

${ }^{4}$ Enfermeira. Doutora em Medicina Tropical. Professora Adjunto da Pontifícia Universidade Católica de Goiás. Goiânia, GO, Brasil. E-mail: cidagi00@gmail.com.

${ }^{5}$ Enfermeiro, Doutor em Ciências da Saúde. Professor Adjunto da Universidade Federal de Goiás. Goiânia, GO, Brasil. E- mail: marcosdeminas@yahoo.com.br.

${ }^{6}$ Enfermeira, Doutora em Ciências da Saúde. Professora Adjunta da Universidade Federal de Goiás. Goiânia, GO, Brasil. E- mail: karlacaetano@gmail.com.

${ }^{7}$ Enfermeira, Doutora em Medicina Tropical. Professora Aposentada da Universidade Federal de Goiás. Goiânia, GO, Brasil. E-mail: minamisava@gmail.com.

${ }^{8}$ Enfermeira, Doutora em Biologia Parasitária. Professora Associada da Universidade Federal de Goiás. Goiânia, GO, Brasil. E-mail: sheila.fen@gmail.com.

${ }^{9}$ Enfermeira, Doutora em Ciências da Saúde. Professora Adjunta da Universidade Federal de Goiás. Goiânia, GO, Brasil. E- mail: marcia.fen@gmail.com.

Recebido: 26/02/2016.

Aceito: $21 / 03 / 2017$

Publicado: $14 / 07 / 2017$

\section{RESUMO}

Objetivou investigar e comparar o conhecimento sobre saúde sexual e reprodutiva, e fontes de informação, entre adolescentes de escolas públicas de Goiânia-Goiás. Estudo de corte transversal realizado com 2449 escolares. Os dados do questionário auto aplicável foram analisados pelo Statistical Package Social Science, versão 13.0. As diferenças entre as proporções foram analisadas pelos testes de $\chi^{2} \mathrm{e}$ nível de significância $(p<0,05)$. Observou-se diferença estatística entre os sexos considerando o conhecimento sobre Infecções Sexualmente Transmissíveis e métodos de prevenção às IST e contracepção $(p<0,000)$, também, adolescentes do sexo masculino apresentaram maior risco de exposição a relações sexuais sem preservativo $(p<0,000)$. Em relação à aquisição de métodos preventivos para IST e contracepção, mulheres demonstraram mais conhecimento de locais de acesso aos dispositivos, bem como buscavam em variadas fontes, informações acerca de conteúdos relacianados à saúde sexual e reprodutiva. Conclui-se que adolescentes do sexo masculino apresentaram maior perfil de vulnerabilidade social e individual.

Descritores: Saúde Sexual e Reprodutiva; Saúde do Adolescente; Vulnerabilidade em Saúde.

\section{INTRODUÇÃO}

A adolescência trata-se de um período complexo, com transformações físicas, cognitivas, sociais e psicológicas resultantes de ajustamentos às construções históricas e sociais. Dentre as
Oliveira PC, Pires LM, Junqueira ALN, Vieira MAS, Matos MA, Caetano KAA, et al. Conhecimento em saúde sexual e reprodutiva: estudo transversal com adolescentes. Rev. Eletr. Enf. [Internet]. 2017 [acesso em:___;19:a17. Disponível em: http://dx.doi.org/10.5216/ree.v19.39926.

transformações físicas a puberdade caracteriza-se como um importante fenômeno biológico, que se inicia 
por mudanças hormonais dando início a um novo ciclo de vida e experiências, traduzidas na afetividade e nos comportamentos sexuais ${ }^{(1,2)}$.

Mediante as mudanças no qual adolescentes vivenciam como a menarca, espermarca, sexarca, alterações físicas, desenvolvimento de caracteres sexuais secundários, alterções psicológicas, muitos não se encontram preparados para lidar com as transformações, o que os coloca sob o risco à problemas psicológicos e estress. Estudo atual aponta que a família, a sociedade e a cultura desempenham funções preditoras no comportamento sexual na adolescência. Neste contexto, práticas sexuais tornam-se objeto de preocupação, uma vez que adolescentes assumem o risco de transmissão de Infecções Sexualmente Transmissíveis (IST), e gestação precoce ${ }^{(2)}$.

Segundo a Pesquisa Nacional de Saúde do Escolar (PeNSE, 2016) a iniciação sexual de adolescentes ocorre na faixa etária de 13 a 15 anos, especialmente no sexo masculino. Destes aproximadamente $60 \%$ fizeram uso de preservativo na sexarca e esse percentual se mantem para o uso do preservativo na última relação sexual. Em relação ao uso de métodos contraceptivos, este mesmo estudo aponta que $70 \%$ dos adolescentes entre 16 e 17 anos já utilizaram algum método contraceptivo ${ }^{(3)}$.

Segundo dados recentes do Boletim Epidemiológico sobre HIV/Aids, no país houve um significativo aumento de taxa de detecção de HIV entre adolescentes com mais de 15 anos. Em um período de 10 anos, entre 2006 e 2015, a taxa de deteç̧ão triplicou sendo que, no ano de 2015 houve 6,9 casos em indivíduos entre 15 e 19 anos em cem mil habitantes. Neste quadro preocupante, o sexo masculino ganha evidência. Evidenciado pelo aumento $70 \%$ a mais de casos entre homens quando comparado a mulheres na faixa etária entre 13 e 19 anos $^{(4)}$.

Assim, conhecimentos e comportamentos sexuais inseguros de adolescentes, impulsionados por conjunturas sociais têm contribuído para a fragilização de toda uma geração de adolescentes e jovens. Portanto, a abordagem do conhecimento em saúde sexual e reprodutiva tornou-se importante tema de saúde pública e tema de debates com necessidades de estratégias intersetoriais para o enfrentamento desta problemática $^{(5)}$

Ainda, tendo em vista a relevância a despeito da temática, ainda há poucos estudos de base populacional que avaliam a relação do conhecimento em saúde sexual e reprodutiva, incluindo as Infecções Sexualmente Transmissíveis (IST) e os fatores relacionados ao comportamento sexual por sexo no grupo de adolescentes.

Nesse sentido, intervenções educacionais poderão ser foco de investimentos ao passo que estudiosos, profissionais de saúde e sobretudo os pais compreendam as expressões da sexualidade na adolescência e a abordem nos contextos em que vivem. Assim, tais percepções contribuirão no planejamento de ações e implementação de políticas que proporcionem o desenvolvimento físico e social saudável do adolescente ${ }^{(2)}$.

Portanto este estudo objetivou investigar e comparar o conhecimento em saúde sexual e reprodutiva, e fontes de informação de adolescentes de escolas públicas.

\section{METODOLOGIA}


Estudo analítico de corte transversal realizado no período de agosto de 2012 a setembro de 2013 com adolescentes escolares de Goiânia. O município está localizado na região central do Brasil, capital do estado de Goiás. A população elegível do estudo constituiu-se por adolescentes escolares de 12 a 18 anos que frequentavam as sete Instituições Públicas de Ensino Básico (IPEB), área de abrangência do Centro de Saúde da Família (CSF), circunvizinhas ao Distrito Sanitário da Região Leste (DSL) da capital. Ressaltamos que essas sete escolas possuíam alunos na faixa etária do estudo.

Do total de matriculados, 2875 indivíduos eram elegíveis, porém 2449 participaram da pesquisa. Foram excluídos aqueles que, embora desejassem participar, não devolveram o Termo de Consentimento Livre e Esclarecido (TCLE) assinado pelo seu responsável legal. Assim da população alvo do estudo alcançamos $85 \%$ da população. Portanto nosso estudo foi representativo para a região escolhida do município. A Figura 1 apresenta o processo de seleção das instituições, recrutamento e seleção dos sujeitos.

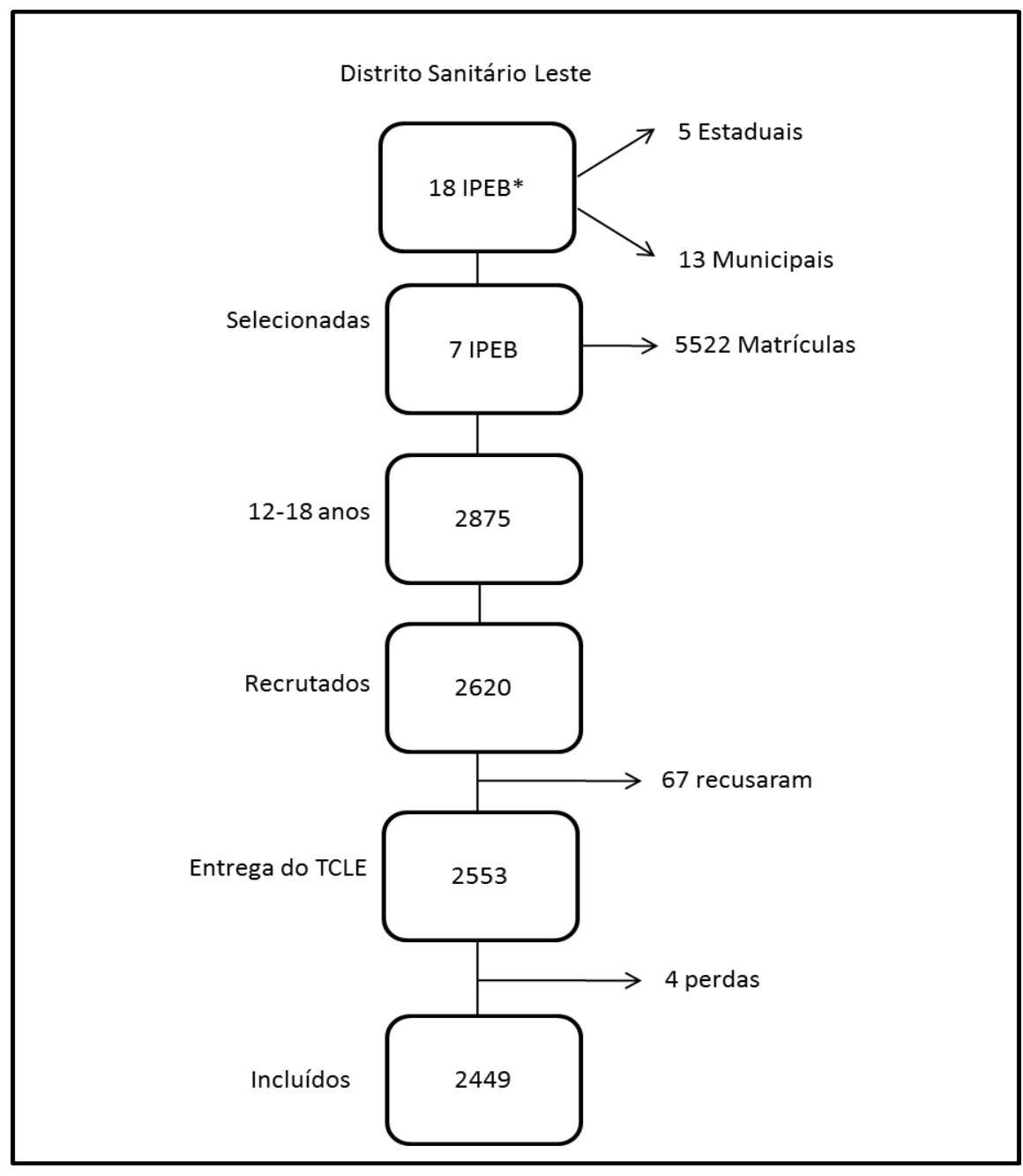

Figura 1: Seleção e recrutamento dos escolares. Goiânia, GO, Brasil, 2012.

A coleta de dados aconteceu por meio de um instrumento anônimo, autoaplicável, de múltipla escolha contendo perguntas sobre conhecimentos e fontes de informação, previamente validado por três pesquisadores com expertise na temática. Visando minimizar vieses de respostas, os auxiliares de pesquisa 
foram capacitados para a coleta dos dados. Em relação a um melhor conforto e garantia da privacidade, os participantes responderam ao questionário em carteiras individualizadas dispostas em amplas salas.

As questões do questionário continha sete questões de dados sóciodemográficos (idade, sexo, cor autodeclarada, instituição de ensino, atividades laborais, com quem residiam e renda familiar), questões que abrangiam informações gerais sobre os conhecimentos e percepções sobre as IST (IST conhecidas, métodos contraceptivos conhecidos, prevenção de IST e situações em que o adolescente assumiria relação sexual insegura). Outras questões investigaram as formas de aquisição de métodos contraceptivos e as formas de acesso as informações sobre prevenção de IST e contracepção.

Utilizou-se para a análise dos dados o software Statistical Package Social Science (SPSS) versão 13. As diferenças entre as proporções foram analisadas pelos testes de $\chi^{2}$ aceitando como nível de significância $(p<0,05)$.

A pesquisa atendeu aos requisitos da Resolução 196/96, legislação vigente na época do estudo, e foi aprovada sob Protocolo do Comitê de Pesquisa Médica Humana e Animal do Hospital das Clínicas da Universidade Federal de Goiás UFG - CEP 058/2009.

\section{RESULTADOS}

Do total de adolescentes escolares investigados ( $n=2449), 52,7 \%$ eram do sexo feminino e $47,3 \%$ do sexo masculino. A média de idade dos adolescentes foi de 14,7 anos (DP: 1,74) (dados não apresentados na tabela). A cor auto declarada predominante foi à parda (59,4\%). Em relação a conformação da família, 55\% referiram residir com os pais, seguidos por $33 \%$ que residiam apenas com a mãe e 9,6\% residiam em famílias compostas por outros arranjos familiares, como por avós e tios. A metade dos adolescentes referiu renda familiar inferior a um salário mínimo.

A Tabela 1 mostra o conhecimento e comportamentos de risco dos adolescentes investigados sobre infecções sexualmente transmissíveis, de acordo com o sexo. Verificou-se diferença estatística entre os sexos, em relação às IST, evidenciando o sexo feminino com maior conhecimento. 
Tabela 1: Conhecimentos sobre saúde sexual e reprodutiva de adolescentes escolares, segundo sexo - Goiânia, Brasil Central, 2012

\begin{tabular}{|c|c|c|c|c|c|c|c|}
\hline \multirow{2}{*}{ Variáveis } & \multirow{2}{*}{$\begin{array}{l}\text { Feminino } \\
(n=1291)\end{array}$} & \multicolumn{3}{|c|}{ Masculino } & \multirow{2}{*}{$\begin{array}{c}\text { Total } \\
(n=2449)\end{array}$} & \multirow{2}{*}{$\%$} & \multirow{2}{*}{$p$} \\
\hline & & $\%$ & $(n=1158)$ & $\%$ & & & \\
\hline \multicolumn{8}{|l|}{ IST conhecidas } \\
\hline Hepatite B & 824 & 63,8 & 612 & 52,8 & 1436 & 58,6 & 0,001 \\
\hline AIDS** & 1217 & 94,3 & 1045 & 90,2 & 2262 & 92,4 & 0,001 \\
\hline Sífilis & 612 & 47,4 & 434 & 37,5 & 1046 & 42,7 & 0,000 \\
\hline Herpes & 607 & 47 & 435 & 37,6 & 1042 & 42,6 & 0,000 \\
\hline $\mathrm{HPV}^{* * *}$ & 445 & 34,5 & 297 & 25,6 & 742 & 30,3 & 0,000 \\
\hline Cancro & 213 & 16,5 & 192 & 16,6 & 405 & 16,5 & 0,800 \\
\hline Donovanose & 73 & 50,7 & 73 & 60,3 & 146 & 60 & 0,400 \\
\hline Nenhuma & 28 & 20,2 & 42 & 30,6 & 70 & 30 & 0,030 \\
\hline \multicolumn{8}{|l|}{ SI: 59} \\
\hline \multicolumn{8}{|l|}{ Como se previne as IST** } \\
\hline Preservativo em todas relações sexuais & 1146 & 88,8 & 1022 & 88,3 & 2168 & 88,5 & 0,900 \\
\hline Evitar parceiros do mesmo sexo & 103 & 80 & 143 & 12,4 & 246 & 10 & 0,001 \\
\hline Ter apenas um parceiro & 495 & 38,4 & 235 & 20,3 & 730 & 29,8 & 0,001 \\
\hline \multicolumn{8}{|l|}{ SI: 67} \\
\hline \multicolumn{8}{|c|}{ Situação em que assumiria relação sexual sem preservativo } \\
\hline Parceria fixa & 300 & 23,2 & 363 & 31,4 & 663 & 27,1 & 0,000 \\
\hline Pessoa conhecida & 27 & 2,1 & 105 & 9,1 & 132 & 5,4 & 0,000 \\
\hline Sem o preservativo no momento & 40 & 3,1 & 154 & 13,3 & 194 & 7,9 & 0,000 \\
\hline Nunca & 833 & 64,5 & 469 & 40,5 & 1302 & 53,2 & 0,000 \\
\hline \multicolumn{8}{|l|}{ SI: 235} \\
\hline \multicolumn{8}{|l|}{ Métodos contraceptivos conhecidos } \\
\hline Contraceptivos Hormonais & 1100 & 85,2 & 718 & 62,0 & 1818 & 74,2 & 0,000 \\
\hline Preservativo masculino & 1093 & 84,7 & 955 & 82,5 & 2048 & 83,6 & 0,090 \\
\hline Métodos naturais & 468 & 36,2 & 250 & 21,6 & 718 & 29,3 & 0,000 \\
\hline SI: 254 & & & & & & & \\
\hline
\end{tabular}

SI: Sem Informação; *IST: Infecção Sexualmente Transmissível; **AIDS: Síndrome da Inumo deficiência Adquirida; ***HPV: Papiloma Vírus Humano.

Quanto às formas de prevenção, o uso do preservativo em todas as relações sexuais não mostrou diferença significante entre os sexos. Já em relação a evitar parceiros do mesmo sexo e ter apenas um parceiro, houve significância, com maior proporção entre o sexo masculino e feminino, respectivamente.

Todas as variáveis investigadas frente à situação de provável relação sexual sem o preservativo demonstraram significância entre os sexos, sendo mais prevalentes entre as adolescentes. Metade dos escolares assinalou não assumir relação sexual sem o uso do preservativo, sendo 64,5\% para o sexo feminino e $40,5 \%$ sexo masculino.

As análises que apresentaram significância demonstraram que o sexo feminino possuía maior conhecimento sobre métodos contraceptivos hormonais e naturais. Já o preservativo masculino não apresentou significância estatística, visto que ambos os sexos já o conheciam $(p=0,09)$. Os achados estão apresentados na Tabela 1.

Ainda, diferença foi observada nas formas de adquirir os métodos de contracepção, sendo a farmácia, Unidade Básica de Saúde e parceria sexual, os mais apontados entre ambos os sexos. Os resultados ainda evidenciaram que os adolescentes buscavam informações em instituições de saúde e ensino, com o (a) namorado (a), com os amigos e os pais. Entretanto a atitude em buscar informações sobressaiu para o sexo feminino, em todas as variáveis $(p<0,001)$. A Tabela 2 evidencia nossos achados em relação à formas de 
aquisição de contraceptivos e fontes de informação sobre saúde sexual e reprodutiva.

Tabela 2: Formas de aquisição de preservativos e fontes de informação sobre sexualidade entre adolescentes escolares, segundo sexo - Goiânia, Brasil Central, 2012

\begin{tabular}{|c|c|c|c|c|c|c|c|}
\hline \multirow{2}{*}{ Variáveis } & \multicolumn{2}{|l|}{ Feminino } & \multirow{2}{*}{$\begin{array}{c}\text { Masculino } \\
(n=1158)\end{array}$} & \multicolumn{3}{|c|}{ Total } & \multirow{2}{*}{$p$} \\
\hline & $(n=1291)$ & $\%$ & & $\%$ & $(n=2449)$ & $\%$ & \\
\hline \multicolumn{8}{|c|}{ Forma de aquisição de métodos contraceptivos } \\
\hline Farmácia & 1014 & 78,6 & 836 & 72,2 & 1850 & 75,6 & 0,000 \\
\hline Unidade Básica de Saúde & 772 & 59,8 & 567 & 49,0 & 1339 & 54,7 & 0,000 \\
\hline Parceria Sexual & 138 & 10,7 & 57 & 4,9 & 195 & 80,0 & 0,000 \\
\hline Amigos & 88 & 6,8 & 85 & 7,3 & 173 & 70,1 & 0,400 \\
\hline Pais & 150 & 11,6 & 106 & 9,2 & 256 & 10,5 & 0,080 \\
\hline \multicolumn{8}{|l|}{ SI: 304} \\
\hline \multicolumn{8}{|c|}{ Fontes de informação e comunicação em sexualidade } \\
\hline Instituições de saúde e ensino & 1030 & 79,8 & 723 & 62,4 & 1753 & 71,6 & 0,000 \\
\hline Mídia/TV/Revista/Internet & 877 & 67,9 & 754 & 65,1 & 1631 & 66,6 & 0,700 \\
\hline Amigos & 745 & 57,7 & 590 & 51,0 & 1335 & 54,5 & 0,001 \\
\hline Pais & 567 & 43,9 & 357 & 30,8 & 924 & 37,7 & 0,000 \\
\hline Namorado (a) & 313 & 24,2 & 211 & 18,2 & 524 & 21,4 & 0,001 \\
\hline SI: 119 & & & & & & & \\
\hline
\end{tabular}

SI: Sem Informação

\section{DISCUSSÃO}

A temáticas em saúde sexual e reprodutiva deveriam ser abordadas de forma cotidiana por profissionais das áreas da saúde e educação junto à adolescentes. Apesar de o tema ser considerado simples e cotidiano, o conhecimento de adolescentes acerca da temática ainda apresenta-se incipiente, como demonstrado no grupo estudado, o que pode contribuir para comportamentos inadequados inseguros e práticas sexuais de risco.

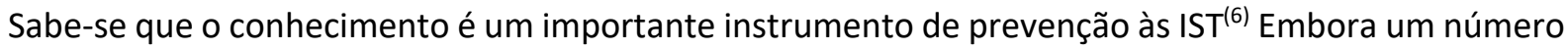
significativo de escolares do presente estudo ter reportado algum nível de conhecimento sobre as IST, somente a AIDS e hepatite B foram relatados por mais da metade dos participantes, sendo o sexo feminio o que demonstrou maior conhecimento sobre às IST, e a diferença entre os sexos apresentou significância estatística referente à hepatite B, aids, sífilis, herpes e HPV. Entretanto, em relação à percentagem de respostas preocupa-nos o desconhecimento de ambos os sexos, quanto às outras infecções que também apresentam relevantes complicações sexuais e reprodutivas na população jovem, como a sífilis, herpes e Papiloma Vírus Humano (HPV). Tal achado ratifica que o déficit de conhecimento desses adolescentes pode levá-los a adotar comportamentos sexuais inseguros desencadeando diversas vulnerabilidades com consequências negativas futuras como iniciação sexual precoce, aquisição de IST, ocorrência de gravidez e problemas sociais, como evasão e abandono escolar ${ }^{(7,8)}$.

Os respondedores do sexo masculino possivelmente se equivocaram em relação a pergunta sobre prevenção de IST quando assinalaram "evitar parceiros do mesmo sexo" como forma para prevenção, independentemente da adesão ao preservativo, dado que apresentou significância estatística. Deste modo possivelmente houve um viez de resposta, o que nos mostra uma interpretação sobre a cultura machista, 
ainda bastante presente no imaginário das pessoas, especialmente nessa população. Ainda, as adolescentes consideraram "ter um único parceiro" como método de prevenção às IST, fenômeno similar a outros estudos $^{(7,9-11)}$.

Ressalta-se que observamos uma importante parcela de adolescentes reconhecia o uso do preservativo em todas as relações sexuais como a principal forma para prevenir gestações indesejadas e IST e ainda foi o método mais conhecido por ambos os sexos, o que, aparentemente, demonstra a aceitação desse método por esse grupo populacional. Entretanto estes dados não amenizam a preocupação diante o apontamento de práticas sexuais desprotegidas quando em relações sexuais com parcerias fixas, pessoas conhecidas e por último se não dispusessem do preservativo no momento da relação sexual.

Observou-se ainda no estudo que meninas tinham melhor conhecimento sobre métodos contraceptivos, e a diferença foi estatisticamente significativa. Esta constatação também foi evidenciada em outros estudos $^{(5,12)}$. Entretanto, apesar das adolescentes apresentarem melhor conhecimento em relação às IST e métodos de prevenção/contracepção, as mesmas encontram-se em uma situação de grande vulnerabilidade em decorrência de seu comportamento sexual desprotegido, creditando confiança nas parcerias sexuais $^{(13)}$.

Assim sendo, inferimos que independente do conhecimento dos adolescentes sobre IST e métodos de prevenção/contracepção, apenas o conhecimento não seja decisivo no ato sexual seguro. Nossos resultados ressaltam a relevância da reformulação de ações e políticas moldadas ao perfil do adolescente atual, que se difere dos jovens das primeiras iniciativas públicas de prevenção de IST/Aids, que apontavam parcerias fixas como medidas de prevenção. Tais iniciativas deverão abordar questões sobre homofobia e parceiro sexual fixo, contribuindo para relações sociais e afetivas mais saudáveis ${ }^{(10)}$.

As diferências encontradas entre os sexos, evidenciando o sexo feminino como maior detentor de conhecimento podem ser explicados pela culturas ocidental e brasileira que responsabilizam à figura feminina o cuidado com a saúde e bem estar da família bem como pelas consequências da vivencia da sexualidade, como riscos à aquisição de infecções transmitidas pela via sexual e possível gravidez ${ }^{(10,12,13)}$, resultados encontrados também em outros países ${ }^{(7,14,15)}$.

Em relação às formas de aquisição de métodos contraceptivos e fontes de informação, igualmente as diferenças entre os sexos evidenciou o sexo feminino. Dentre as formas apontadas como meios de obtenção dos métodos contraceptivos, foram apontadas a farmácia, a Unidade de Saúde e a/o parceiro. Destacamos que a aquisição de método preventivos, especialmente de barreira em estabelecimentos comerciais, como nas farmácias não garante a obtenção de um produto seguro, pois há uma grande oferta de insumos atrativos como a variedades de cores, sabores e por vezes fora da data de validade além de que, no ato da compra, não recebem as orientações para o uso, potencializando, então, o uso e a adesão frágil aos métodos com consequente abandono. Sendo que essa população pode ter melhor acesso a estes insumos nas unidades de saúde entregues por profissionais de saúde com potencial mais seguro para informações. Com a implantação e implementação de projetos governamentais o Projeto Saúde e Prevenção nas Escolas (SPE) é considerado 
como importante estratégia para a garantia de informações e oferta de insumos nas instituições de ensino, principalmente o preservativo masculino, com a adaptação anatômica já disponível para a população sexualmente ativa, em particular para os adolescentes. Contudo essa discussão deve ser melhor abordada entre profissionais de saúde, educação, gestores e pais ${ }^{(16)}$.

Todas as variáveis analisadas sofrem influencias dos veículos de informação bem como da qualidade das informações que os adolescentes acessam ou possuem contato. Novamente o sexo feminino sobressaiu na diferença estatística entre os sexos referente à busca por informações sobre saúde sexual e reprodutiva ${ }^{(9)}$. As adolescentes do estudo acessam mais fontes de informação, principalmente, em instituições de saúde e ensino, mídias e amigos, dados que diferem de outras pesquisas que evidenciam como principais fontes de informação a família e os amigos ${ }^{(17)}$ e a família e o professor $^{(18)}$.

Segundo dados da PeNSE (2015) 70\% de adolescentes entre 13 e 15 anos e de 76\% de adolescentes entre 16 e 17 anos receberam orientações sobre prevenção de gravidez indesejada. Entretanto quando se refere às IST o percentual de acesso à informação aumenta para aproximados 80\% de 13 a 15 anos de idade e $84 \%$ dos escolares do grupo etário de 16 a 17 anos. No referido estudo a informação sobre a aquisição de preservativos não obteve igual sucesso, sendo que aproximados 60\% (13-15 anos) e de 75\% (16-17) ${ }^{(4)}$.

Ressalta-se que os dados sobre fontes de informações continuam sendo indispensáveis estratégias de educação por promover a socialização entre adolescentes com discussão em grupos, rodas de conversa, experiências compartilhadas, fortalecimento de uma rede de apoio entre família, saúde, escola e comunidade de forma a compartilhar os conhecimentos, dúvidas e tabus, ainda comuns nessa população ${ }^{(17,19,20)}$, com especial atenção ao grupo mas jovem ${ }^{(5)}$ fortalescendo o protagonismo dos mesmos na promoção de ações educativas em saúde sexual e reprodutiva ${ }^{(21)}$.

Nesse sentido, torna-se imperativo a elaboração e efetivação de estratégias de discussão rotineira acerca das questões relativas às vulnerabilidades, gênero e sexualidade no ambiente escolar. Para tanto, os investimentos da gestão pública de todas as esferas governamentais, juntamente com os profissionais da saúde e educação devem ser direcionadas para impactar o cotidiano dos adolescentes no sentido de que as informações sobre as IST sejam de fato transformadas em saberes e práticas seguras com conscientização para tomada de decisões mais assertivas ${ }^{(22)}$.

Em nosso estudo as diferenças observadas entre os sexos ratificaram o sexo feminino com melhores níveis de conhecimento e percepção de risco, resultado que está em consonância com a pesquisa nacional de conhecimentos atitudes e práticas da população brasileira ${ }^{(23)}$. Todavia, os dados dessa investigação são preocupantes à medida que estes adolescentes são de instituições da rede básica de ensino que possuem Centros de Saúde da Família próximos á instituição de ensino e o Programa Saúde na Escola já implantado, entretanto, deve-se direcionar um olhar cuidadoso para os educadores, pois, apesar da temática em questão ser relevante para o desenvolvimento integral e saudável de adolescente, muitos professores ainda apresentam dificuldades em abordar o tema ${ }^{(24)}$.

Assim, este estudo ao abordar conhecimentos e percepções comportamentais sobre saúde sexual e 
reprodutiva de adolescentes permitiu observar possíveis determinantes de vulnerabilidade individual às IST(25), como o provável sexo desprotegido em razão de ter parceiro fixo ou com pessoa conhecido e baixo conhecimento de métodos contraceptivos e de IST, em especial pelo pelos indivíduos do sexo masculino ${ }^{(16)}$. A partir dos resultados faz-se necessário a articulação da políticas públicas de saúde do adolescente com as políticas de atenção à saúde do homem a fim de minimizar as ideias de subalternidade feminina e empoderar os adolescentes do sexo masculino acerca das vulnerabilidades observadas.

Nosso estudo possui algumas limitações. Embora todos os cuidados adotados no momento da aplicação do questionário, possíveis vieses decorrentes da concepção de estudos de corte transversal podem ocorrer como, a falta de cuidados nas respostas como responsabilidade/seriedade no ato das respostas, viés de memória, pressa em terminar de responder, autocensura e receio de que autoridades escolares e/ou pais pudessem ter acesso as respostas. Em contrapartida o questionário autoaplicado permitiu que os participantes respondessem com mais autonomia.

\section{CONCLUSÕES}

Apesar de todo aparato tecnológico e da globalização da informação e das políticas públicas de saúde voltadas para os adolescentes escolares, o conhecimento equivocado ou insuficiente acerca dos vários aspectos sobre saúde sexual e reprodutiva ainda é um problema emergente, principalmente entre adolescentes do sexo masculino. Tais fatores evidenciam a cultura hegemônica masculina que limita a responsabilização da saúde sexual e reprodutiva aos meninos.

Os resultados deste estudo nos possibilitou conhecer que adolescentes do sexo feminino apresentaram melhor conhecimento sobre métodos contraceptivos e IST, em relação ao sexo masculino. Esse dado pode sinalizar que adolescentes do sexo masculino apresentaram maior perfil de vulnerabilidade individual em relação ao sexo feminino, evidenciando a necessidade de consolidação de políticas educacionais e de saúde, assim como do adolescente e do homem com foco nas representações de gênero, visando construtos que subsidiem estes adolescentes a assumirem atitudes e práticas sexuais mais seguras e livres de ideias preconcebidas em relação ao gênero desigual.

Pensar em estratégias de intervenções ou investigações de natureza qualitativa a serem trabalhadas no futuro com a mesma população é salutar, pois respostas obtidas em estudos quantitativos podem não responder a subjetividade emergente da temática do estudo. Este estudo apresentou vantagens pois podese oportunizar a participação do adolescente nas discussões de temas de interesse deles próprios, rompendo barreiras como a timidez e os tabus, próprios dessa fase da vida, além de ser um estudo de base populacional representativo, abranger grupos de distintas idades por sexo e fazer uma avaliação de comportamentos de riscos com perguntas iguais para os dois sexos. Tais características, pouco exploradas em outros estudos puderam ser analisadas.

Vislumbramos a necessidade da implementação e acompanhamento de ações de saúde e prevenção nas escolas, como o envolvimento de pais de adolescentes, capacitação de professores e profissionais de 
saúde, especialmente o enfermeiro, e atuação das Instituições de Ensino Superior no contexto escolar com projetos de extensão e pesquisa no campo da saúde sexual e reprodutiva, a fim de minimizar as diversas vulnerabilidades que os adolescentes estão expostos.

\section{REFERÊNCIAS}

1. World Health Organization. Helth Topics. Adolescent health [Internet]. 2015 [acesso em: 05 Fev. 2017]. Disponível em: http://who.int/topics/adolescent health/en/.

2. Kar SK, Choudhary A, Singh AP. Understanding normal developmet of adolescent sexuality: A bumpy ride. J Hum Reprod Sci [Internet]. 2015;8(2):70-4. Disponível em:

http://www.ncbi.nlm.nih.gov/pmc/articles/PMC4150140/?report=printable.

3. Instituto Brasileiro de Geografia e Estatística - IBGE. Pesquisa Nacional de Saúde do Escolar. Rio de Janeiro; 2016.

4. Ministério da Saúde; Secretaria de Vigilância em Saúde. Boletim Epidemiológico - Aids e DST. Brasilia; 2016.

5. Gonçalves H, Machado E, Soares A, Camargo-Figueira F. Inicio da vida sexual entre adolescentes (10 a 14 anos) e comportamentos em saúde. Rev Bras Epidemiol. 2015;18(1):25-41.

6. Castro EL, Caldas TA, Morcillo AM, Pereira EM de A, Velho PENF. O conhecimento e o ensino sobre doenças sexualmente transmissíveis entre universitários. Aware Educ regarding Sex Transm Dis among Undergrad students [Internet]. 2016;21(6):1975-84. Disponível em:

http://search.ebscohost.com/login.aspx?direct=true\&db=poh\&AN=115984378\&site=ehost-live.

7. Madkour AS, Farhat T, Halpern CT, Godeau E, Gabhainn SN. Early adolescent sexual initiation as a problem behavior: A comparative study of five nations. J Adolesc Heal [Internet]. Elsevier Ltd; 2010;47(4):389-98. Available from: http://dx.doi.org/10.1016/j.jadohealth.2010.02.008.

8. Langille $D$, Asbridge $M$, Flowerdew G, Allen M. Associations of sexual risk-taking with having intercourse before 15 years in adolescent females in Cape Breton, Nova Scotia, Canada. Sex Heal. 2010;7(2):199-204.

9. Cruzeiro A, Souza L, Silva R, Pinheiro R, Rocha C, Horta B. Comportamento sexual de risco: fatores associados ao numero de parceiros sexuais e ao uso de preservativos em adolescentes. Ciênc Saúde Coletiva. 2010;15(1):1149-58. 10. Valim EMA, Dias FA, Simon CP, Almeida DV de, Rodrigues MLP. Utilização de preservativo masculino entre adolescentes de escolas públicas na cidade de Uberaba (MG), Brasil: conhecimentos e atitudes. Cad Saúde Coletiva. 2015;23(1):44-9.

11. Miranda AAM, Silva CGO, Thimoteo GM, Assis LF, Del'Duca A, Carvalho AR de, et al. Conhecimentos acerca de dst/aids e métodos contraceptivos dos discentes dos cursos técnicos integrados do IF sudeste MG - Campus Juiz de Fora, Brasil. Multiverso. 2016;1(1):25-36.

12. Malta DC, Silva MAI, Mello FCM De, Monteiro RA, Porto DL, Sardinha LMV, et al. Saúde sexual dos adolescentes segundo a Pesquisa Nacional de Saúde dos Escolares. Rev Bras Epidemiol. 2011;14(1):147-56.

13. Tronco CB, Dell'aglio DD. Caracterização do Comportamento Sexual de Adolescentes : Iniciação Sexual e Gênero. Rev Interinstitucional Psicol. 2012;5(2):254-69.

14. Kachur R, Mesnick J, Liddon N, Kapsimalis C, Habel M, David-Ferdon C, et al. Adolescents, Technology and Reducing Risk for HIV, STDs and Pregnancy. Atlanta: Centers for Disease Control and Prevention; 2013.

15. Peltzer K. Early sexual debut and associated factors among in-school adolescents in eight African countries. Acta Pediatr. 2010;99(8):1242-7.

16. Russo K, Arreguy ME. Projeto "Saúde e Prevenção nas Escolas": Percepções de professores e alunos sobre a distribuição de preservativos masculinos no ambiente escolar. 2015;25(2):501-23.

17. Silva AT, Jacob MHVM, Hirdes A. Conhecimento de adolescentes do ensino médio sobre DST. Aletheia. 2015;46(October):43-9.

18. Oliveira NDP, Béria JU, Schermann LB. Sexualidade na adolescência : um estudo com escolares da cidade de Manaus / AM. Aletheia. 2014;43-44:129-46.

19. Bahia S, Trindade JP. O potencial das tecnologias educativas na promoção da inclusão: três exemplos. Educ Formação Tecnol [Internet]. 2010;3(1):96-110. Disponível em: http://eft.educom.pt/index.php/eft/article/view/128. 
20. Griffin KW, Botvin GJ. Evidence-Based Interventions for Preventing Substance Use Disorders in Adolescents. Child Adolesc Psychiatr Clin N Am. 2010;19(3):505-26.

21. Iossi MAS, Mello DF, Carlos MO. Adolescents as protagonists in health educational activities at school. 2010;2(2):3-8.

22. Bezerra M, Seifert C. Uma apresentação reflexiva da sexualidade humana no espaço escolar: da negação a emancipação erótica. Na Scien cult. Paranaíba. 2010;2(1):287-93.

23. Ministério da Saúde. Programa Nacional de DST e AIDS. Pesquisa de Conhecimentos, Atitudes e Práticas na População Brasileira. Brasilia; Ministério da Saúde, 2011.

24. Rufino CB, Pires LM, Oliveira PC, Souza SMB, Souza MM. Educação sexual na prática pedagógica de professores da rede básica de ensino. Rev Eletrônica Enferm. 2013;15(4):983-91.

25. Ayres J, Calazans G, Filho HS, França-Júnior I. Risco, vulnerabilidade e práticas de prevenção e promoção da saúde. In: Campos GWS, Bonfim JRA, Minayo MCS et al. Tratato de Saúde Coletiva. 2‥ São Paulo: Hucitec; 2012. p. 399-442. 